\title{
Extinction partially reverts structural changes associated with remote fear memory
}

\author{
Gisella Vetere, ${ }^{1,2}$ Leonardo Restivo, ${ }^{1,3}$ Giovanni Novembre, ${ }^{1}$ Massimiliano Aceti, ${ }^{2,4}$ \\ Massimo Lumaca, ${ }^{1}$ and Martine Ammassari-Teule ${ }^{1,2,5}$ \\ ${ }^{1}$ Cell Biology and Neurobiology Institute, C.N.R.-National Research Council of Italy, 00143 Rome, Italy; ${ }^{2}$ IRCCS Santa Lucia \\ Foundation, 00143 Rome, Italy
}

\begin{abstract}
Structural synaptic changes occur in medial prefrontal cortex circuits during remote memory formation. Whether extinction reverts or further reshapes these circuits is, however, unknown. Here we show that the number and the size of spines were enhanced in anterior cingulate $(\mathrm{aCC})$ and infralimbic (ILC) cortices $36 \mathrm{~d}$ following contextual fear conditioning. Upon extinction, aCC spine density returned to baseline, but the enhanced proportion of large spines did not. Differently, ILC spine density remained elevated, but the size of spines decreased dramatically. Thus, extinction partially erases the remote memory network, suggesting that the preserved network properties might sustain reactivation of extinguished conditioned fear.
\end{abstract}

The observation that dendritic spine growth develops in the anterior cingulate cortex during the formation of enduring fear memory (Restivo et al. 2009) has put forward the idea that in addition to changes in the strength and efficacy of existing synapses, large-scale modifications in prefrontal cortical connectivity, including the anterior cingulate cortex (aCC) and the infralimbic cortex (ILC), also underlie systems memory consolidation (Frankland et al. 2004). Nonetheless, how long the content of a remote memory and the wiring diagram of its structural support remain stable over time is not well understood. Memories can be rearranged through further experience depending on their tendency to be retrieved and reconsolidated with different content. This process encompasses a variety of operations, since a memory can be simply updated to maintain its relevance (Winocur et al. 2007; Lee 2009) or extinguished when novel information conflicts with an earlier formed representation (Quirk and Mueller 2008). Extinction, which largely depends on ILC (Quirk et al. 2000), can therefore be viewed as an extreme form of memory rearrangement, and whether it implicates that the network supporting the original representation is erased or further remodeled is undetermined.

To investigate the structural changes underlying remote extinction, independent groups of 9-wk-old male C57BL/6J@Ico mice (Charles River Italia [Calco, Como]) were trained or pseudotrained for contextual fear conditioning (CFC) and returned to the chamber, where no footshock was delivered either $36 \mathrm{~d}$ later, or from day 31 to day 36 to generate extinction (Fig. 1A). CFC consisted of one single session of $7 \mathrm{~min}$, during which each mouse was placed in a transparent Plexiglas cage $(28 \times 28 \times 10 \mathrm{~cm})$ with a removable grid floor made of stainless-steel rods. After $120 \mathrm{sec}$ of free exploration, the mouse was exposed to a series of five nonsignaled footshocks (duration: $2 \mathrm{sec}$; intensity: $0.7 \mathrm{~mA}$; 60 -sec apart) delivered through the grid floor. Pseudo-trained mice were treated identically, except that they were not shocked.

Present addresses: ${ }^{3}$ Program in Neurosciences and Mental Health, The Hospital for Sick Children, Toronto, Ontario M5G 1X8, Canada; ${ }^{4}$ Neuroscience Department, The Scripps Research Institute, 130 Scripps Way, Jupiter, FL 33458, USA.

${ }^{5}$ Corresponding author.

E-mail martine.teule@cnr.it.

Article is online at http://www.learnmem.org/cgi/doi/10.1101//m.2246711.
Contextual fear remote memory tests were run by placing the mice back into the conditioning chamber for $5 \mathrm{~min}$ in the absence of any footshock either $36 \mathrm{~d}$ after the conditioning (consolidation group: CONS, $n=6$ ) or the pseudo-conditioning (PSEUDO group, $n=5$ ), or for 7 min from day 32 to day 35 with a 5-min test on day 36 (extinction group: EXT, $n=6$ ). Behavior during conditioning and testing was recorded by means of a video camera mounted $60 \mathrm{~cm}$ above the ceiling of the cage and connected to a computer equipped with the Ethovision software (Noldus). The time spent freezing (absence of all but respiratory movements) was used to score fear memory. Results were expressed as means \pm SEM, and a one-way ANOVA with group (PSEUDO, CONS, EXT) as main factor was used to compare freezing scores recorded during training and testing. A one-way ANOVA with days as main factor was also carried out in the EXT group to estimate the decrease in freezing across the five extinction sessions. Data are shown in Figure 1B that outline the presence of intense freezing during the test in the CONS mice and the extinction of freezing in the EXT mice. Specifically, during training the shocked mice (CONS and EXT) exhibited stronger freezing than the PSEUDO mice (effect of group, $F_{(2,16)}=19.26, P<0.0001$; PSEUDO vs. any shocked group, $P<0.001)$. During extinction, freezing decreased progressively in the EXT group re-exposed to the context on post-training days $32-35$ (effect of days, $F_{(3,21)}=46.47, P<0.0001$ ). During the test, only the CONS mice still showed freezing to the context (effect of group, $F_{(2,16)}=313.35, P<0001$; CONS vs. PSEUDO and EXT, $P<0.001$ ). Importantly, the amount of freezing in the extinguished mice was indistinguishable from the amount of freezing measured in the pseudo-trained mice (EXT vs. PSEUDO, $P=0.87)$.

On day 37, mice were deeply anesthetized with chloral hydrate $(400 \mathrm{mg} / \mathrm{kg}$ i.p.) and perfused transcardially with a solution of $0.9 \%$ saline. Brains were dissected and impregnated using a Golgi-Cox solution according to the method described by Glaser and Van der Loos (1981). They were first immersed in the GolgiCox solution at room temperature for $6 \mathrm{~d}$, transferred to a $30 \%$ sucrose solution for $2 \mathrm{~d}$, and then sectioned using a vibratome. Coronal sections $(100-\mu \mathrm{m}$ thick) were mounted on gelatinized slides, stained according to the method described by Gibb and Kolb (1998), and coverslipped with Permount. Spine density was analyzed on pyramidal neurons located in layers II/III of the 
A
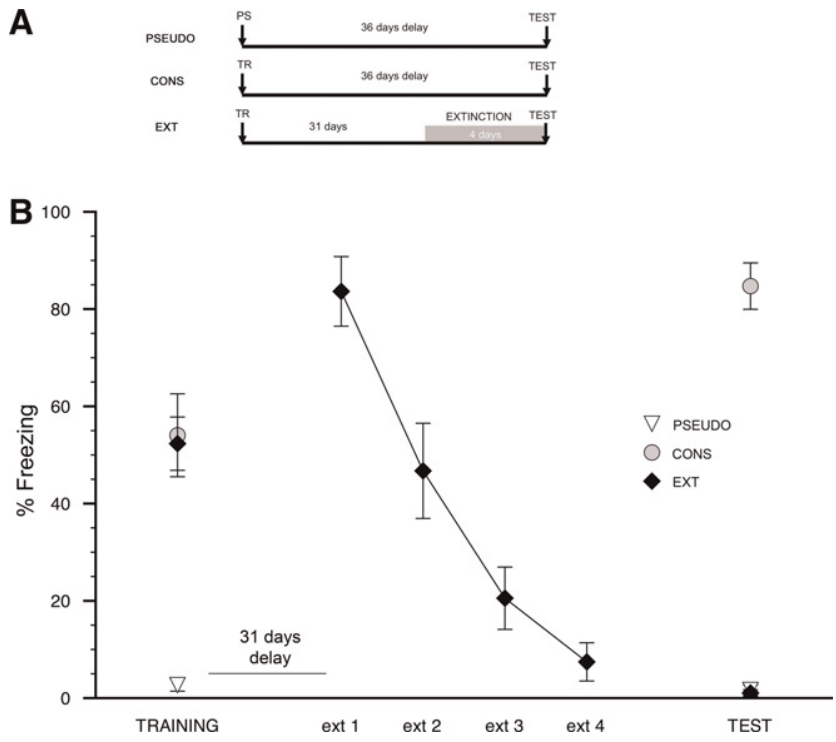

Figure 1. Mice show intense freezing upon remote consolidation and virtually no freezing upon extinction. (A) Experimental design. (B) Mice subjected to contextual fear conditioning (CONS: gray circles) show a robust fear response to the context compared with pseudo-conditioned mice (PSEUDO: white triangles) during the test run $36 \mathrm{~d}$ later; mice exposed to the chamber in the absence of footshock from day 31 to 35 (EXT: black diamonds) show a gradual reduction of their fear response until it becomes virtually absent on day 36 . Comparison of freezing scores during the test, effect of group, $F_{(2,16)}=313.35, P<0001$; CONS vs. any group, $P<0.001$.

anterior cingulate (aCC) and infralimbic (ILC) cortices defined according to the Franklin and Paxinos (2001) mouse atlas. Measurements were performed on apical dendrites that were previously found to undergo the stronger CFC-induced structural remodeling (Restivo et al. 2009). Neurons were identified with a light microscope (Leica DMLB) under low magnification (20X/ NA 0.5) in coronal sections of each region of interest. Three neurons showing at least fourth-order branches in the apical dendrite compartment were selected within each hemisphere in each region. Since no significant interhemispheric difference was found, measurements were pooled, giving a final sample of six neurons per region for each animal. The total number of neurons analyzed was the following: aCC-PSEUDO: $n=27$; aCC-CONS: $n=33$; aCC-EXT: $n=34$; ILC-PSEUDO: $n=30$; ILC-CONS: 35 ; ILC-EXT: $n=35$ ).

Spine counts and measurements were performed on secondary and tertiary branches of apical dendrites of anterior cingulate cortex and infralimbic cortex pyramidal neurons in layers II/III.

Spines were counted using an optical microscope (Leica DMLB) with a video camera (Qimaging Qicam Fast1394) connected to the microscope. Neurons were drawn and analyzed using the Neurolucida software. On each neuron, five 30-100- $\mu \mathrm{m}$ apical dendritic segments were randomly selected. In some cases, segments were from the same branch (Leuner et al. 2003). Segments were sampled $50 \mu \mathrm{m}$ away from soma in order to exclude the spine-depleted zone that arises from the cell body. Only protuberances with a clear connection of the head of the spine to the shaft of the dendrite were counted as spines. Since this method has proven to provide reliable results (Horner and Arbuthnott 1991), no attempt was made to introduce a correction factor for hidden spines. As no difference in spine counts was observed between secondary and tertiary branch segments for each group, data were pooled to generate the final spine density counts, expressed as number of spines per micrometer. The spine raw data were subsequently averaged for a neuron mean. For spine size measurements, selected dendrites were imaged with a video camera (Qimaging Qicam Fast1394) connected to the optical microscope (Leica DMLB) using the Neurolucida software. Images were acquired as a series of optical z-sections at $0.4-\mu \mathrm{m}$ increments using an oil immersion 100X objective (NA 1.25). They were exported to NIH ImageJ software for analysis to create a z stack. Head diameters were measured only on spines appearing continuous with their parent dendritic shaft. The number of spines measured in each group ranged from 917 to 1000. Spine morphology analysis was performed blind to experimental conditions. Differences in spine density between PSEUDO, CONS, and EXT were analyzed in each region by means of a one-way ANOVA with group as main factor. In the same regions, cumulative frequencies of spine head diameters measured in PSEUDO, CONS, and EXT were compared using first the normal distribution Kolmogorov-Smirnov (K-S) fitting test and then the K-S twosample tests. Post hoc analyses were performed where necessary using the Fisher's protected least significant differences test. The data are shown in Figure 2.

After the remote memory test, group differences in spine density were found on apical neuron dendrites in both the aCC $\left(F_{(2,91)}=16.67, P<0.0001\right)$ (Fig. $\left.2 \mathrm{~A}, \mathrm{~B}\right)$ and the ILC $\left(F_{(2,97)}=\right.$ 9.63, $P<0.001$ ) (Fig. 2D,E). In the aCC, post-hoc analyses revealed that spines were more numerous in CONS mice than in PSEUDO $(P<0.0001)$ and EXT $(P<0.0001)$ mice, the last difference depending on the reset of spine density to pseudoconditioning values following extinction (EXT vs. PSEUDO, $P=$ 0.39). Conversely, ILC spines were more numerous in CONS and EXT mice than in PSEUDO mice (CONS vs. PSEUDO: $P<0.01$ and EXT vs. PSEUDO: $P<0.001$ ), indicating that their number remained elevated upon extinction (EXT vs. CONS, $P=0.28$ ) (Fig. 2D,E). Measurements of spine head diameters in the aCC across conditions revealed a significant shift of the curve to the right in CONS mice, but also in EXT mice, compared with PSEUDO mice $(P<0.001$ for each comparison), revealing that encoding of an aversive contextual representation increased spine size on the majority of neurons examined, and showed that this increase persisted upon extinction (Fig. 2C). For the ILC, the same significant shift to the right was observed for the curve of CONS mice compared with PSEUDO mice $(P<0.0001)$, but a shift to the left of the EXT curve, even outreaching the PSEUDO mice curve $(P<0.0001)$, pointed to a recrudescence of small spines upon extinction in this region (Fig. 2F).

Thus, although the same large-scale variations in neuronal connectivity occurred in the aCC and the ILC during the formation of remote contextual fear memory, these regions were diversely remodeled upon extinction, therefore suggesting that they contributed differently to the modification of the original fear representation.

In agreement with data showing that the aCC and the ILC are both involved in remote fear memory formation (Frankland et al. 2004), we observed that mice tested for remote consolidation $36 \mathrm{~d}$ after CFC showed a robust fear response to the context that was associated with a consistent increase in spines $(\sim 12 \%)$ in the apical compartment of aCC and ILC pyramidal neuron dendrites. CFC also increased the size of spines in these regions, supporting the view that large spines provide structural storage sites for longterm associative memory (Matsuzaki et al. 2004; Bourne and Harris 2007). Indeed, spine enlargement has been shown to correlate with enhanced synaptic strength during long-term potentiation (LTP) in immature (Gu et al. 2010) and mature (Bozdagy et al. 2010) hippocampal synapses. There is also evidence that the proportion of large mushroom spines is selectively increased on CA1 neurons $48 \mathrm{~h}$ after water-maze training (Hongpaisan and Alkon 


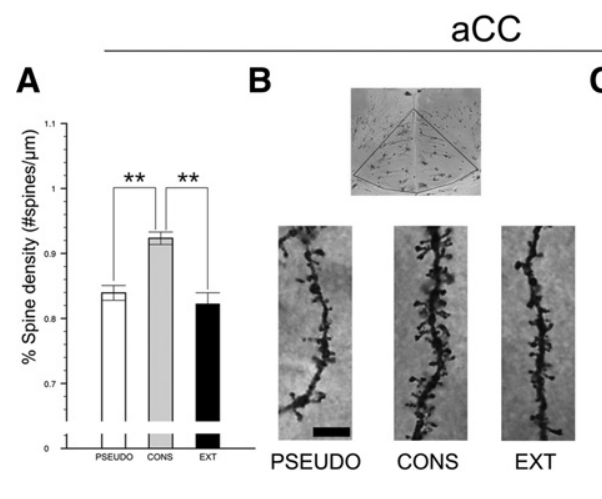

\section{C}

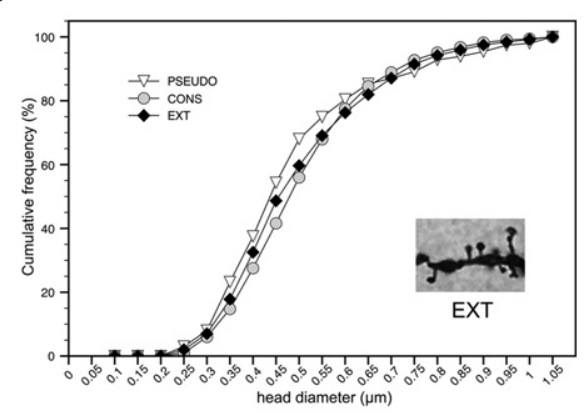

ILC

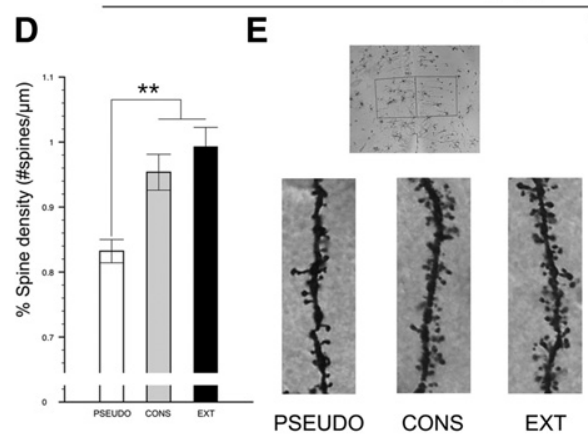

$\mathbf{F}$

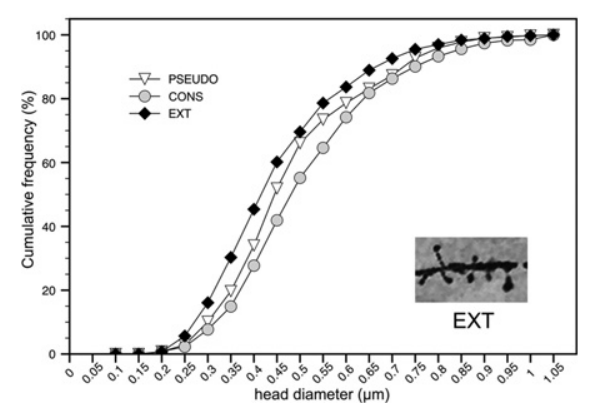

Figure 2. Remote consolidation and extinction diversely alters spine density and spine size in anterior cingulate (aCC) and and infralimbic (ILC) cortices. ( $A$ ) aCC spine density measured on apical dendrites was increased in consolidated (CONS: gray bars) relative to pseudo-conditioned (PSEUDO: white bars) mice, but returned to baseline in the extinguished (EXT: black bars) mice (effect of group, $F_{(2,91)}=$ 16.67, $P<0.0001$; CONS vs. any group, $P<0.0001$; EXT vs. PSEUDO, $P=0.39)$. (B) Representative images of Golgi-impregnated aCC region and photomicrographs of aCC dendrite segments depicting group differences in spine density. Scale bar, $5 \mu \mathrm{m}$. (C) Cumulative frequency distribution curves for aCC spine head diameters and representative images of spines from the EXT group. Cumulative frequency data show a significant shift of the curve to the right in trained (CONS and EXT) relative to nontrained (PSEUDO) mice ( $P<0.001$ for each comparison), indicating larger spine head diameters in the majority of the sample neurons in CONS and EXT. (D) ILC spine density was increased in CONS and EXT mice relative to PSEUDO mice (effect of group, $F_{(2,97)}=9.63, P<0.001$; CONS vs. PSEUDO, $P<0.01$ and EXT vs. PSEUDO, $P<0.001$; EXT vs. CONS, $P=0.28)$. ( $E$ ) Representative images of Golgi-impregnated ILC region and photomicrographs of ILC dendrite segments depicting group differences in spine density. Scale bar, $5 \mu \mathrm{m}$. $(F)$ Cumulative frequency distribution curves for ILC spine head diameters and representative images of spines from EXT group. Compared with the curve of PSEUDO mice, a significant shift to the right was observed for the curve of CONS mice $(P<0.0001)$, and to the left for the curve of EXT mice $(P<0.0001)$ indicating that, compared with the values recorded following pseudo-training, the spine head size was increased upon remote consolidation and decreased upon extinction in the majority of the spines examined. $\left(^{* *}\right) P<0.01$.

2007), indicating a direct relationship between the morphology and the function of spines. In these reports, however, large spines were observed shortly after LTP or learning, and exclusively in the potentiated or the primary recruited neurons. Our findings reveal that spine enlargement promoted by CFC can be detected over considerably longer periods of time (36 d post-training) on neurons laying in remotely recruited cortical regions.

We then found that extinction partially reverted consolidation-induced structural changes by segregating rearrangements in the density or in the size of spines according to the region. Repeated exposure to the chamber in the absence of footshock gradually diminished the fear response to the context until freezing was virtually absent and the density of aCC spines returned to pseudo-conditioning values (Fig. 2A,B). However, the size of spines remained large (Fig. 2C). This observation is in line with data showing that while most experience-dependent new synapses are transient, only a fraction of synapses associated with spine enlargement are stabilized (Holtmaat et al. 2005). This means that it is not the number, but the size of spines that needs to be kept up for maintaining the trace in a latent state and permit its reactivation long after training (Hongpaisan and Alkon 2007). Thus, our observation that the number of large spines remains elevated also after extinction suggests that those spines might also sustain the reactivation of fear memory even after the fear response has been extinguished. In contrast, ILC spine density remained elevated upon extinction, while the size of spines decreased dramatically (Fig. 2D,E). Data showing that the aCC and the ILC are concomitantly activated during remote memory consolidation, but only lesions to (Takehara et al. 2003) or inactivation of (Frankland et al. 2004) the aCC are effective in blocking remote retrieval, implicate that the ILC contributes to the formation of trace, but is not essential for its reactivation. Thus, once this region has been engaged in memory formation, it might be potentially recruited to serve other functions. Among those, converging evidence indicates that the ILC is crucial for fear extinction. For example, ILC lesions prevent extinction of fear responses (Quirk et al. 2000; Chang and Maren 2010), fear extinction enhances ILC neuronal activity (Milad and Quirk 2002), and activation of the ILC in a fear context accelerates fear extinction (Thompson et al. 2010). Indeed, the size of ILC spines upon extinction (Fig. 2F), which is decreased even below the levels observed upon pseudo-training, indicates that new protrusions were formed in this region. Our hypothesis is that these recently formed synaptic contacts account for a reshaping of ILC connections coherent with the novel function in which this region is involved.

Collectively, our data show that fluctuations in spine density and spine shape occur in remotely recruited cortical regions during both the formation and the extinction of contextual fear memories. The observation that remote extinction resets the density, but not the size of aCC spines, while it left unchanged the density, but not the size of ILC spines, provides evidence that the neuronal network supporting the original representation is only partially remodeled in the extinguished mice. In particular, the persistence of spine enlargement in the aCC upon extinction suggests that the role of large spines in long-term memory might be to warrant the reactivation of both consolidated and extinguished fear representations. Future studies will therefore focus on the proportion and persistence of large aCC spines in protocols producing abnormally resistant fear memories (Debiec and Ledoux 2004; Quirk et al. 2010) and on the possibility that treatment targeting the molecular specificity of large spines (Bourne and Harris 2007) might be the most effective in erasing these pathological memories. 


\section{Acknowledgments}

This work was supported by the Santa Lucia Foundation and Regione Lazio Bioscience Technology Program. We thank Hélène Marie and Gavin Rumbaugh for comments on earlier drafts of this manuscript.

\section{References}

Bourne J, Harris KM. 2007. Do thin spines learn to be mushroom spines that remember? Curr Opin Neurobiol 17: 381-386.

Bozdagi O, Wang XB, Nikitczuk JS, Anderson TR, Bloss EB, Radice GL, Zhou Q, Benson DL, Huntley GW. 2010. Persistence of coordinated long-term potentiation and dendritic spine enlargement at mature hippocampal CA1 synapses requires N-cadherin. J Neurosci 30: 9984-9989.

Chang CH, Maren S. 2010. Strain difference in the effect of infralimbic cortex lesions on fear extinction in rats. Behav Neurosci 124: 391-397.

Debiec J, Ledoux JE. 2004. Disruption of reconsolidation but not consolidation of auditory fear conditioning by noradrenergic blockade in the amygdala. Neuroscience 129: 267-272.

Frankland PW, Bontempi B, Talton LE, Kaczmarek L, Silva AJ. 2004. The involvement of the anterior cingulate cortex in remote contextual fear memory. Science 304: 881-883.

Franklin KBJ, Paxinos G. 2001. The mouse brain in stereotaxic coordinates, 2nd ed. Academic Press, San Diego, CA.

Gibb R, Kolb B. 1998. A method for vibratome sectioning of Golgi-Cox stained whole rat brain. J Neurosci Methods 79: 1-4.

Glaser EM, Van der Loos H. 1981. Analysis of thick brain sections by obverse-reverse computer microscopy: Application of a new, high clarity Golgi-Nissl stain. J Neurosci Methods 4: 117-125.

Gu J, Lee CW, Fan Y, Komlos D, Tang X, Sun C, Yu K, Hartzell HC, Chen G, Bamburg JR, et al. 2010. ADF/cofilin-mediated actin dynamics regulate AMPA receptor trafficking during synaptic plasticity. Nat Neurosci 13: $1208-1215$.

Holtmaat AJ, Trachtenberg JT, Wilbrecht L, Shepherd GM, Zhang X, Knott GW, Svoboda K. 2005. Transient and persistent dendritic spines in the neocortex in vivo. Neuron 45: 279-291.
Hongpaisan J, Alkon DL. 2007. A structural basis for enhancement of long-term associative memory in single dendritic spines regulated by PKC. Proc Natl Acad Sci 104: 19571-19576.

Horner CH, Arbuthnott E. 1991. Methods of estimation of spine densityare spines evenly distributed throughout the dendritic field? J Anat 177: 179-184.

Lee JL. 2009. Reconsolidation: Maintaining memory relevance. Trends Neurosci 32: 413-420.

Leuner B, Falduto J, Shors TJ. 2003. Associative memory formation increases the observation of dendritic spines in the hippocampus. J Neurosci 23: 659-665.

Matsuzaki M, Honkura N, Ellis-Davies GC, Kasai H. 2004. Structural basis of long-term potentiation in single dendritic spines. Nature 429: $761-766$.

Milad MR, Quirk GJ. 2002. Neurons in medial prefrontal cortex signal memory for fear extinction. Nature 420: $70-74$.

Quirk GJ, Mueller D. 2008. Neural mechanisms of extinction learning and retrieval. Neuropsychopharmacology 33: 56-72.

Quirk GJ, Russo GK, Barron JL, Lebron K. 2000. The role of ventromedial prefrontal cortex in the recovery of extinguished fear. J Neurosci 20: $6225-6231$.

Quirk GJ, Pare D, Richardson R, Herry C, Monfils MH, Schiller D, Vicentic A. 2010. Erasing fear memories with extinction training. J Neurosci 30: 14993-14997.

Restivo L, Vetere G, Bontempi B, Ammassari-Teule M. 2009. The formation of recent and remote memory is associated with time-dependent formation of dendritic spines in the hippocampus and anterior cingulate cortex. J Neurosci 29: 8206-8214.

Takehara K, Kawahara S, Kirino Y. 2003. Time-dependent reorganization of the brain components underlying memory retention in trace eyeblink conditioning. J Neurosci 23: 9897-9905.

Thompson BM, Baratta MV, Biedenkapp JC, Rudy JW, Watkins LR, Maier SF. 2010. Activation of the infralimbic cortex in a fear context enhances extinction learning. Learn Mem 17: 591-599.

Winocur G, Moscovitch M, Sekeres M. 2007. Memory consolidation or transformation: Context manipulation and hippocampal representations of memory. Nat Neurosci 10: 555-557.

Received April 14, 2011; accepted in revised form June 28, 2011. 


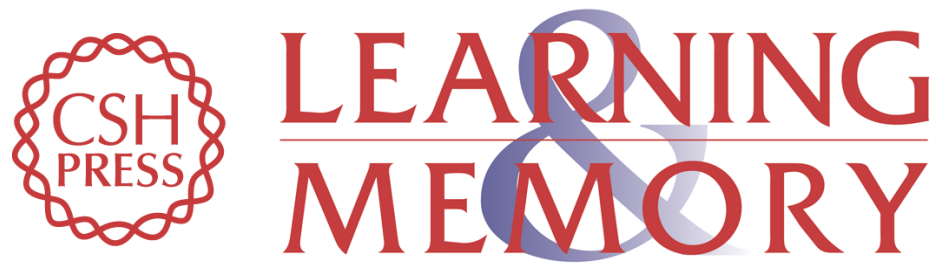

\section{Extinction partially reverts structural changes associated with remote fear memory}

Gisella Vetere, Leonardo Restivo, Giovanni Novembre, et al.

Learn. Mem. 2011, 18:

Access the most recent version at doi:10.1101//m.2246711

References This article cites 22 articles, 9 of which can be accessed free at:

http://learnmem.cshlp.org/content/18/9/554.full.html\#ref-list-1

License

Email Alerting Receive free email alerts when new articles cite this article - sign up in the box at the Service top right corner of the article or click here. 